\title{
Pharmacologic therapy for nonalcoholic steatohepatitis focusing on pathophysiology
}

\section{In Cheol Yoon, Jong Ryeol Eun}

Department of Internal Medicine, Myongji Hospital, Hanyang University College of Medicine, Goyang, Korea

Received: March 20, 2019

Revised: April 4, 2019

Accepted: April 4, 2019

Corresponding author:

Jong Ryeol Eun

Department of Internal Medicine,

Myongji Hospital, Hanyang

University College of Medicine, 55,

Hwasu-ro 14beon-gil, Deogyang-

gu, Goyang 10475, Korea

Tel: +82-31-810-5114

Fax: +82-31-969-0500

E-mail:dreundavis@gmail.com
The paradigm of chronic liver diseases has been shifting. Although hepatitis B and C viral infections are still the main causes of liver cirrhosis and hepatocellular carcinoma (HCC), the introduction of effective antiviral drugs may control or cure them in the near future. In contrast, the burden of nonalcoholic fatty liver disease (NAFLD) has been increasing for decades, and 25 to $30 \%$ of the general population in Korea is estimated to have NAFLD. Over 10\% of NAFLD patients may have nonalcoholic steatohepatitis (NASH), a severe form of NAFLD. NASH can progress to cirrhosis and HCC. NASH is currently the second leading cause to be placed on the liver transplantation list in the United States. NAFLD is associated with obesity, type 2 diabetes, dyslipidemia, and metabolic syndrome. The pathophysiology is complex and associated with lipotoxicity, inflammatory cytokines, apoptosis, and insulin resistance. The only proven effective treatment is weight reduction by diet and exercise. However, this may not be effective for advanced fibrosis or cirrhosis. Therefore, effective drugs are urgently needed for treating these conditions. Unfortunately, no drugs have been approved for the treatment of NASH. Many pharmaceutical companies are trying to develop new drugs for the treatment of NASH. Some of them are in phase 2 or 3 clinical trials. Here, pharmacologic therapies in clinical trials, as well as the basic principles of drug therapy, will be reviewed, focusing on pathophysiology.

Keywords: Drug therapy; Nonalcoholic steatohepatitis; Pathophysiology

\section{Introduction}

Nonalcoholic fatty liver disease (NAFLD) is a chronic disease in which lipid accumulates in hepatocytes, and hepatocyte ballooning is seen pathologically. The spectrum is wide, ranging from simple steatosis to steatohepatitis (NASH), fibrosis, and even cirrhosis [1]. Lipotoxicity is the main pathophysiology associated with NASH initiation and progression [2]. Even though the main lipid is a form of triglyceride (TG), other lipid metabolites also accumulate. Lipid metabolites, such as free cholesterol (FC) and free fatty acids (FFAs), cause apoptosis via up-regulation of the death receptors, sensitizing them to inflammatory cytokines [2] (Fig. 1). Caspases and the Bcl2 families are involved in the apoptotic pathway [3] (Fig. 2). Even hepatocytes, including Kupffer cells, phagocytize apoptotic cell debris. They release transforming growth factor- $\beta$ (TGF- $\beta$ ), the main cytokine of fibrosis, which causes the activation of hepatic stellate cells (HSCs) and more accumulation of lipids in the hepatocytes via Smad2/3 [4] (Fig. 3). Therefore, lipotoxicity and apoptosis may be suitable therapeutic targets for the treatment of NASH.

Endotoxin (lipopolysaccharide) activates intrahepatic macrophages (Kupffer cells) via toll like receptor 4 (TLR4), which then release inflammatory cytokines including $\mathrm{C}$-C motif chemokine ligand 2 (CCL2). C-C motif chemokine receptor 2 


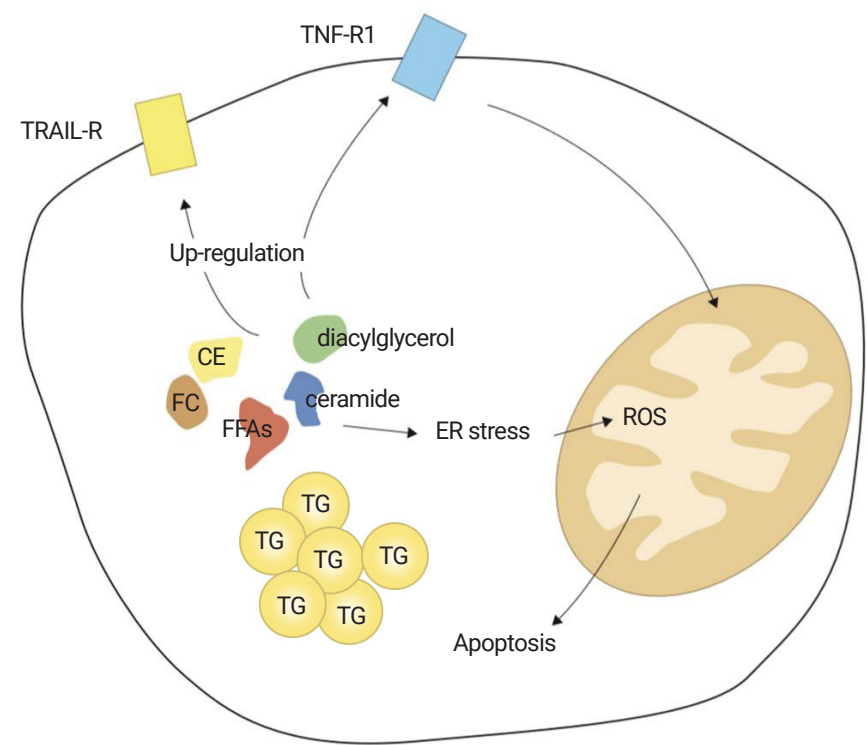

Fig. 1. Lipotoxicity. Although the major form of lipids is $T G$, other lipid metabolites also accumulate: $F F A s, F C$, diacylglycerol, $C E$, and ceramide. "The quality" rather than "quantity" of lipids may contribute to lipotoxicity. Especially, FC and FFAs are important mediators of lipotoxicity. They up-regulate death receptors, then sensitizing them to TNF- $\alpha$. They also trigger ER stress, causing mitochondrial dysfunction and apoptosis (adapted from Okazaki I. [Non-alcoholic steatohepatitis]. Cho YK, Eun JR, translators. Paju: Koonja Publishing Inc.; 2018. p. 152 [5], with permission of Koonja Publishing Inc.). TG, triglyceride; FFA, free fatty acid; FC, free cholesterol; CE, cholesterol ester; TNF- $\alpha$, tumor necrosis factor- $\alpha$; ER, endoplasmic reticulum; TNF-R1, tumor necrosis factor receptor 1; TRAIL-R, TNF-related apoptosis-inducing ligand receptor; ROS, reactive oxygen species.

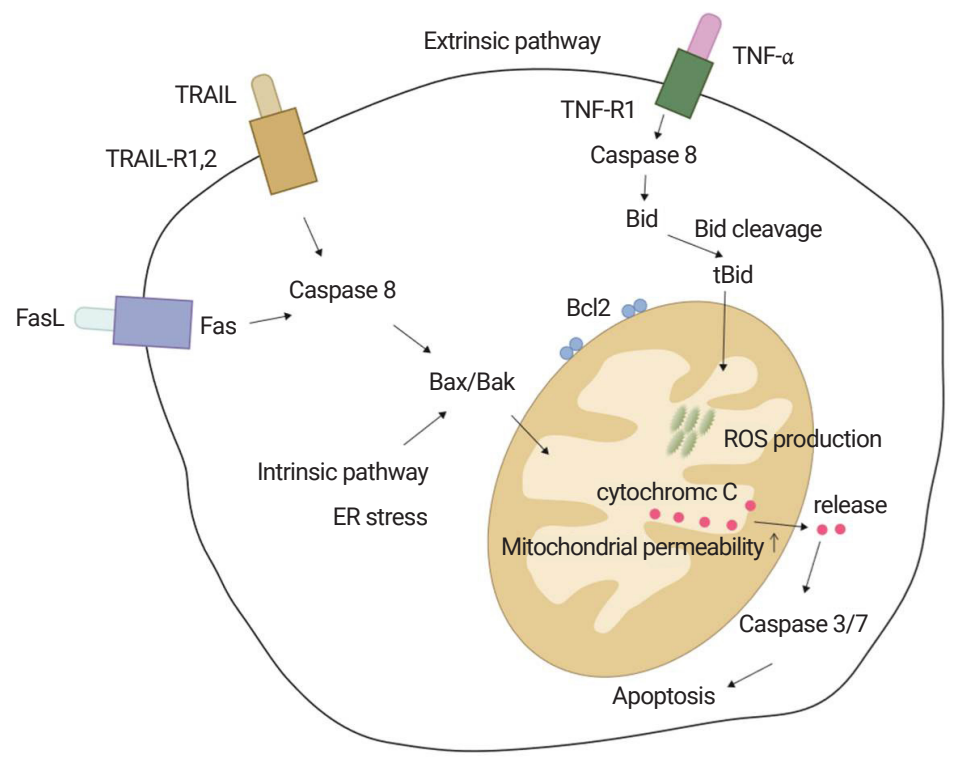

Fig. 2. Basic concept of apoptosis. Apoptosis is a key consequence of cell injury. There are two pathways: extrinsic pathway via deathreceptors, such as the TNF- $\alpha$ receptor, Fas, and TRAIL; and the intrinsic pathway via ER stress. Both pathways go through the mitochondria. Mitochondrial dysfunction is a key event of apoptosis. Caspases are enzymes associated with the apoptotic process. Caspase 8 is an initiator caspase and caspase 3/7 is an effector caspase. The Bcl2 family is also involved with apoptosis. Bid, Bad, Bim, and Bax/Bak are pro-apoptotic and $\mathrm{Bcl} 2$ and $\mathrm{Bcl}-\mathrm{xL}$ are anti-apoptotic. Caspase 8 occurs cleavage of Bid to $\mathrm{Bid}$, leading to mitochondrial permeabilization and the release of cytochrome $C$. Released cytochrome $C$ activates caspase $3 / 7$, the effector caspase, resulting in the final apoptotic morphology (adapted from Okazaki I. [Non-alcoholic steatohepatitis]. Cho YK, Eun JR, translators. Paju: Koonja Publishing Inc.; 2018. p. 151 [5], with permission of Koonja Publishing Inc.). TNF- $\alpha$, tumor necrosis factor- $\alpha$; TRAIL, TNF-related apoptosis-inducing ligand; ER, endoplasmic reticulum; tBid, truncated Bid; ROS, reactive oxygen species. 


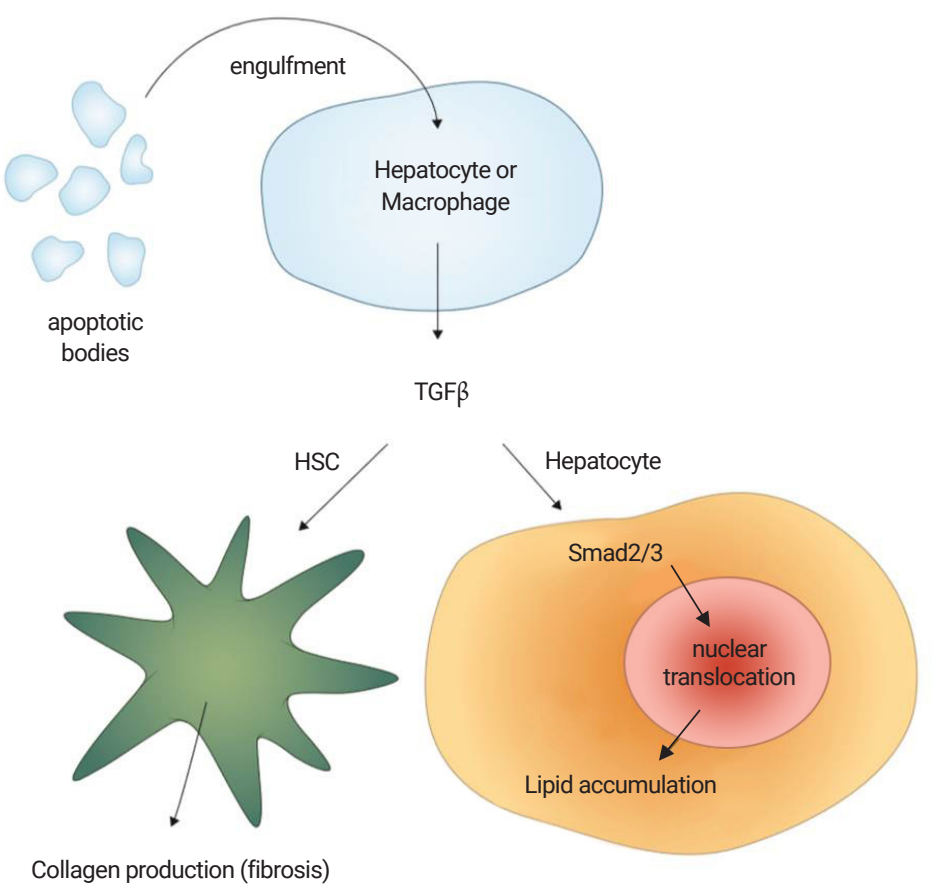

Fig. 3. TGF- $\beta$ signaling in steatohepatitis. Even hepatocytes, as well as Kupffer cells, phagocytize apoptotic cell debris. They release TGF- $\beta$, the main cytokine in fibrosis, which causes the activation of HSCs and more accumulation of lipids in hepatocytes via Smad2/3 (adapted from Okazaki I. [Non-alcoholic steatohepatitis]. Cho YK, Eun JR, translators. Paju: Koonja Publishing Inc.; 2018. p. 154 [5], with permission of Koonja Publishing Inc.). TGF- $\beta$, transforming growth factor- $\beta$; HSC, hepatic stellate cell.

(CCR2)-positive bone marrow (BM)-derived monocytes and HSCs respond to CCL2 and are recruited to the liver [6]. CCR5 is expressed on HSCs and lymphocytes. CCR5 cells infiltration via chemokine-chemokine interaction cause inflammation and fibrosis in the liver [7]. The phase $2 \mathrm{~b}$ CENTAUR trial was performed to evaluate the efficacy of cenicriviroc (CVC), a dual inhibitor of CCR2/CCR5 [8].

Obesity and insulin resistance are the main risk factor for NASH progression, as well as for cardiovascular disease $[9,10]$. Hyperglycemia and hyperinsulinemia increase the expression of connective tissue growth factor and activate the type 1 collagen gene in HSCs [9]. Hyperglycemia and hyperinsulinemia have been associated with hepatocellular carcinoma (HCC) in NASH [10]. Insulin sensitizers, such as thiazolidinediones and metformin, are prescribed for the treatment of type 2 diabetes worldwide [11]. Accordingly, they have been investigated as treatments for NASH [12]. Apoptosis signal-regulating kinase 1 (ASK1) is a key enzyme in insulin resistance and inflammation in NASH. The mitochondrial oxidative stress caused by inflammatory cytokines activates c-Jun-N-terminal kinase (JNK) via ASK1 [13]. The activated JNK interrupts insulin action by serine phosphorylation of the insulin receptor substrate 1 , not tyrosine phosphorylation
[14] (Fig. 4). Selonsertib, an ASK1 inhibitor, improved metabolic parameters in phase 2 clinical trials [14]. Two phase 3 trials are ongoing $[13,14]$.

Cirrhosis is the final event in the fibrotic process. Lysyl oxidaselike 2 (LOXL2) is an enzyme which stabilizes collagen crosslinking. LOXL2 is involved in fibrosis progression, cirrhosis, and even HCC development by directing hepatic progenitor cells toward cholangiocyte differentiation (ductular reaction) [15] (Fig. 5). Simtuzumab, an anti-LOXL2 monoclonal antibody, was tried in clinical trials anticipating an anti-fibrotic effect [16].

Recently, the farnesoid X receptor (FXR), a bile acid synthesis regulator, has gained attention. In the ileum, FXR activates fibroblast growth factor 19 (FGF-19), which increases insulin sensitivity by glucagon-like peptide-1 (GLP-1) activation. Moreover, in the liver, FXR inhibits TG synthesis by inhibiting the sterol regulatory element-binding protein 1c (SREBP-1c) via the short heterodimer partner (SHP), stimulates the $\beta$-oxidation of FFAs by peroxisome proliferator-activated receptors (PPAR)- $\alpha$ activation, and improves glucose homeostasis [17] (Fig. 6). Obeticholic acid (OCA), an FXR agonist, has been tested for the treatment of NASH [18].

Although many drugs are under phase 2 or 3 clinical trials, none have been approved by the Food and Drug Administration (FDA) 


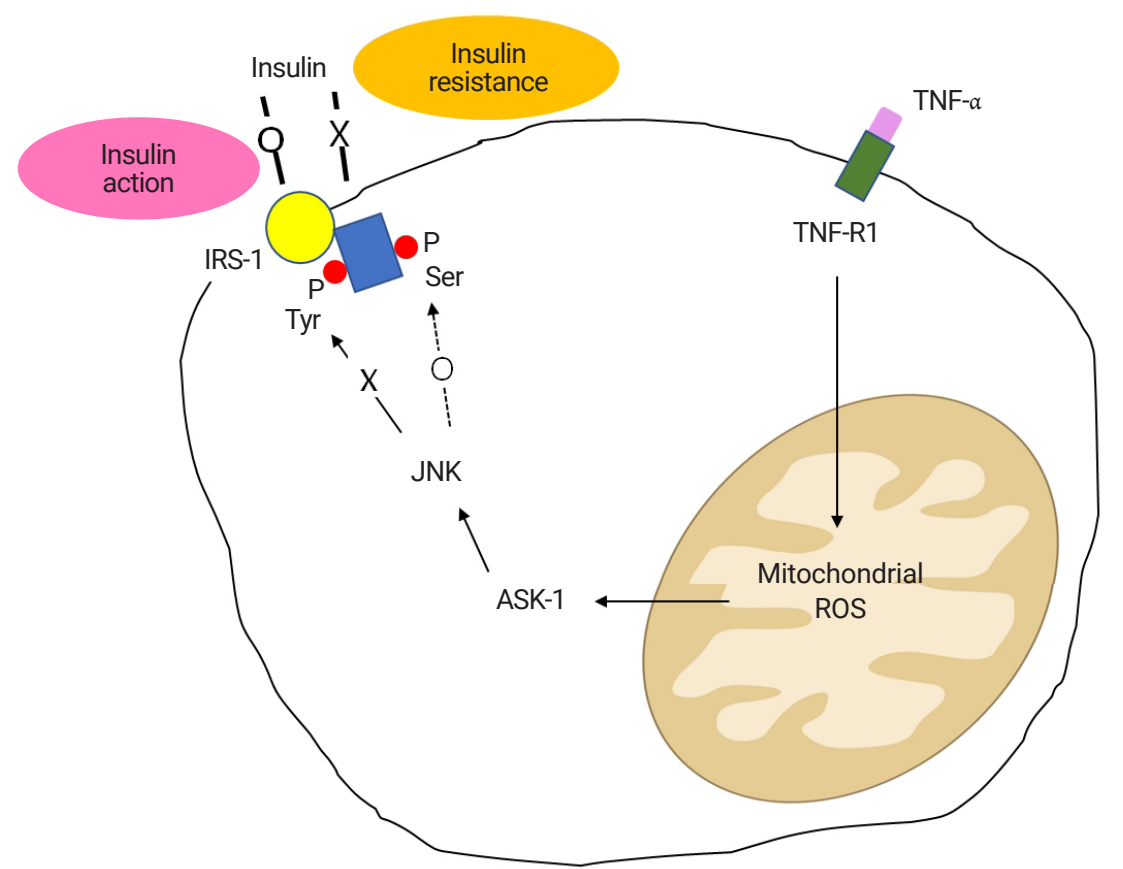

Fig. 4. ASK1 and insulin resistance. The inflammatory cytokine, TNF- $\alpha$, produces mitochondrial ROS, which activates ASK1. ASK1 activates JNK, and subsequently causes serine phosphorylation of IRS-1. Normally, the tyrosine phosphorylation of IRS-1 is a key event in the action of insulin. Impaired tyrosine phosphorylation and increased serine phosphorylation of IRS-1 are associated with insulin resistance. ASK1, apoptosis signal-regulating kinase 1 ; TNF- $\alpha$, tumor necrosis factor- $\alpha$; ROS, reactive oxygen species; JNK, c-Jun-NH2-terminal kinases; IRS-1, insulin receptor substrate-1; ROS, reactive oxygen species.

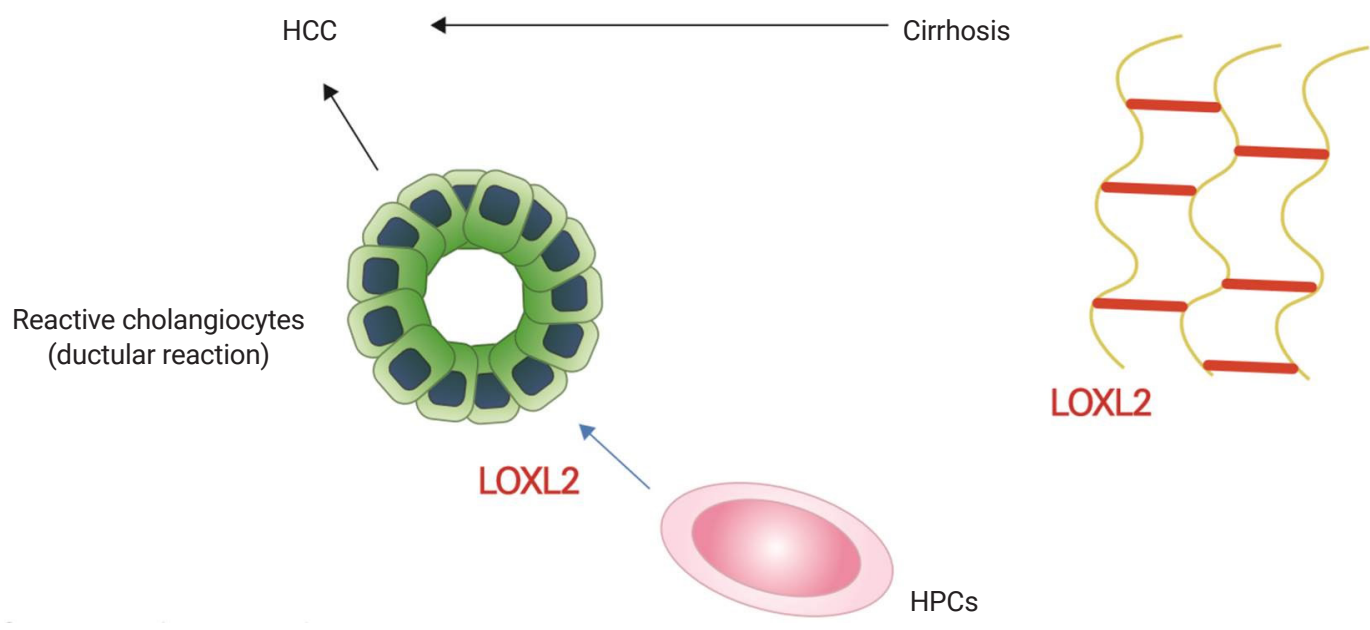

Fig. 5. Lysyl oxidase. The LOX family is composed of four isoforms, LOX and the LOXL1-4. Among them, LOXL2 is a stabilizer of collagen crosslinking. LOXL2 is also expressed in hepatic progenitor cells in fibrotic liver. LOXL2 promotes progenitor cells towards fibrogenic cholangiocytes, suppressing their differentiation into hepatocytes. The ductular reaction characterized by reactive cholangiocytes is associated with hepatocarcinogenesis (adapted from Okazaki I. [Non-alcoholic steatohepatitis]. Cho YK, Eun JR, translators. Paju: Koonja Publishing Inc.; 2018. p. 225 [5], with permission of Koonja Publishing Inc.). LOX, lysyl oxidase; LOXL1-4, LOX-like 1-4; HCC, hepatocellular carcinoma; HPC, hepatic progenitor cell. 


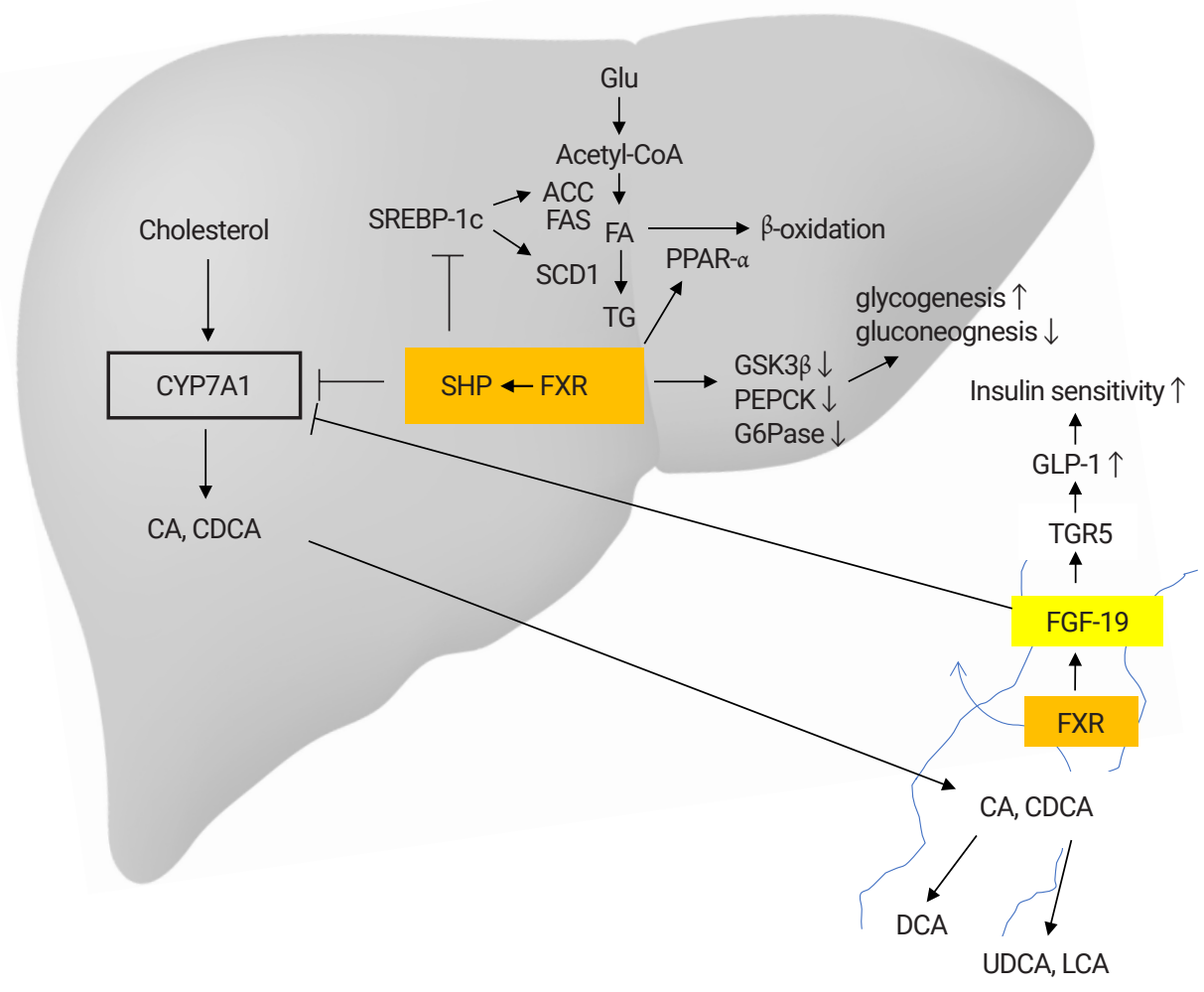

Fig. 6. Farnesoid $X$ receptor. The primary bile acids, $C A$, and CDCA, are synthesized in the liver and secreted into the bile duct. The CYP7A1 is key for this process. Approximately 95\% of the primary bile acids are reabsorbed in the ileum. A small proportion ( $5 \%)$ enters the colon. Here, secondary bile acids are metabolized by intestinal microbiota. The majority of the bile acids are re-used by the enterohepatic circulation and only some are newly-synthesized. The FXR is a key nuclear receptor for bile acid resorption in the ileum. FXR stimulates FGF-19 in the enterocytes, which inhibits CYP7A1, resulting in reduced new bile acid synthesis by negative feedback. The FXR nuclear receptors are also expressed in the liver, adrenal glands and kidneys. In the liver, FXR acts via a SHR. Intrahepatic FXR/SHP represses bile acid synthesis by inhibiting CYP7A1 and TG synthesis by inhibiting SREBP-1c. It also stimulates the $\beta$-oxidation of FFAs by PPAR- $\alpha$ activation, increases glycogenesis and suppresses glycolysis. Intestinal FXR induces FGF-19, which increases insulin sensitivity by GLP-1 activation (adapted from Okazaki I. [Non-alcoholic steatohepatitis]. Cho YK, Eun JR, translators. Paju: Koonja Publishing Inc.; 2018. p. 225 [5], with permission of Koonja Publishing Inc.). CA, cholic acid; CDCA, chenodeoxycholic acid; CYP7A1, cytochrome P450 7A1; FXR, farnesoid X receptor; FGF-19, fibroblast growth factor 19; SHR, short heterodimer partner; SHP, short heterodimer partner; TG, triglyceride; FFA, free fatty acid; PPAR- $\alpha$, peroxisome proliferator-activated receptor- $\alpha$; GLP-1, glucagon-like peptide-1; ACC, acetyl-CoA carboxylase; FAS, fatty acid synthase; SREBP-1, sterol-responsive element binding protein-1; SCD1, steroyl coenzyme A desaturase 1; DCA, deoxycholic acid; UCDA, ursodeoxycholic acid.

to date. This review will cover the basic principles of pharmacologic therapy in practice, and the important results of phase 2 or 3 clinical drug trials, focusing on pathophysiology in detail.

\section{Basic principles of pharmacologic therapy}

To treat underlying associated diseases, such as type 2 diabetes, dyslipidemia, and hypertension.

\section{The use of anti-diabetic drugs in cases of co-morbid type 2 diabetes}

1) Pioglitazone and rosiglitazone
Pioglitazone and rosiglitazone are anti-diabetic drugs of the thiazolidinedione family which stimulate PPAR- $\gamma$, decreasing fatty acid migration into the liver and increasing $\beta$-oxidation by AMPactivated protein kinase (AMPK) activation [19]. Moreover, PPAR- $\gamma$ inhibits HSC activation and increases adiponectin levels $[19,20]$. Randomized controlled trials (RCTs) were conducted based on this background [21]. Insulin resistance and liver enzymes improved during the treatment, but the effect was not sustained after the treatment was stopped. Histologic improvement in steatosis, inflammation, and hepatocellular ballooning was observed. However, fibrosis improvement was not confirmed. Common adverse effects were weight gain and edema. Safety 
concerns regarding myocardial infarction and bladder cancer remain [21].

\section{2) Metformin}

Metformin is a first-line anti-diabetic drug of the biguanide family. Metformin attenuates insulin resistance via activation of the AMPK pathway, hence it increases the uptake of peripheral glucose into the liver, muscle, and adipose tissue. It also suppresses gluconeogenesis in the liver and lipolysis in adipose tissue [21]. A study on metformin showed that the liver enzyme normalization and histologic improvement rates were higher compared to the vitamin E or diet-only groups [22]. Metformin showed a weight-reducing effect and a satisfactory safety profile. Some studies showed the improvement of insulin resistance and aminotransferase levels. However, a recent meta-analysis concluded that metformin did not improve liver histology. Therefore, it is not recommended as first-line therapy for the treatment of NASH patients without diabetes [23].

\section{3) Glucagon-like peptide 1 agonist}

The native GLP-1 agonist increases insulin secretion and suppresses glucagon secretion. It also delays gastric emptying and decreases appetite. However, endogenous GLP-1 is degraded in minutes by dipeptidyl peptidase 4 [24]. Liraglutide, a long-acting GLP-1 agonist, was approved in 2009 as an anti-diabetic drug in obese patients by the effects of weight reduction, pancreatic betacell function improvement, $\mathrm{HbAlc}$ reduction, and beneficial effects on blood pressure. In the LEAN study, liraglutide showed NASH resolution in 39\% (9/23) of the patients, weight reduction and glucose control [24]. In December 2014, liraglutide $\left(\right.$ Saxenda ${ }^{\circledR}$ ) was approved by the FDA for the treatment of obesity (body mass index $[\mathrm{BMI}] \geq 30$, or $\mathrm{BMI} \geq 27$ plus dyslipidemia or type 2 diabetes or hypertension) based on the SCALE study [25]. All GLP-1 agonists are given as injections.

\section{4) Sodium/glucose cotransporter 2 inhibitor}

SGLT2 inhibitors are anti-diabetic drugs which act by increasing the urinary excretion of glucose. They also reduce body weight and blood pressure [26]. Several pilot studies have shown significant reductions in alanine transaminase (ALT), body weight, and the fatty liver index in NAFLD patients. The impact on liver histology was not confirmed [27]. A recent RCT showed that luseogliflozin significantly reduced liver fat deposition, as well as visceral fat, HbAlc, and BMI compared to metformin [28]. In another RCT, ipragliflozin showed body weight and visceral fat reduction compared to pioglitazone [29]. A few pilot studies of SGLT2 inhibitors are ongoing [26].

\section{The use of anti-hyperlipidemic agents in cases of dyslipidemia}

1) Statins

As mentioned in the introduction section, lipotoxicity is the main pathophysiology of NASH initiation and progression. The altered quality of lipids, rather than their quantity, causes lipotoxicity [2]. Altered lipid metabolites up-regulate death receptors, sensitizing them to inflammatory cytokines. They also cause endoplasmic reticulum (ER) stress. Extrinsic and intrinsic stimuli cause mitochondrial oxidative stress, then finally, apoptosis. Moreover, FC accumulation in the HSCs increases TLR4, then activates TGF- $\beta$ via bone morphogenic protein and activin membranebound inhibitor inhibition, which causes a vicious cycle of FC accumulation [30].

Statins have lipid-lowering effects as HMG-CoA reductase inhibitors. They also have anti-oxidant and anti-inflammatory effects [2]. Atorvastatin has been reported to decrease mitochondrial FC and increase glutathione levels [2]. These agents should be considered for the prevention of cardiovascular diseases in NAFLD patients with hypercholesterolemia. Several studies have suggested that statins may improve liver enzymes and histology in patients with NASH. However, no RCTs with histological endpoints have been conducted. Until RCTs with histological endpoints are undertaken, statins are not recommended for the treatment of NASH without dyslipidemia [23].

\section{2) Omega-3 fatty acids}

N-6 fatty acids have inflammatory, and n-3 fatty acids have antiinflammatory action. Moreover, an increased n-6/n-3 fatty acids ratio increased the HCC risk in a NASH mouse model [31]. Omega-3 fatty acids showed improvement in hepatic steatosis, insulin sensitivity, oxidative stress, and anti-inflammatory action in an animal model [32]. Omega-3 fatty acids are currently approved in the United States to treat hypertriglyceridemia [23]. Two large studies failed to show therapeutic benefit in patients with NAFLD/NASH $[33,34]$. Therefore, they are not recommended for the treatment of NAFLD without hypertriglyceridemia [23].

\section{3) Fibrates}

Fibrates, such as bezafibrate or fenofibrate, are extensively used for the treatment of hypertriglyceridemia. But there is little data on NAFLD/NASH.

\section{4) Ezetimibe}

Ezetimibe inhibits the intestinal absorption of luminal cholesterol by binding to the Niemann-Pick C1-like 1 transporter in the membrane of the enterocyte brush border. In an animal model, 
it reduced cholesterol absorption up to 15 to $20 \%$ and hepatic fat content, and improved insulin resistance [35]. But the MOZART RCT trial failed to demonstrate reductions in liver fat contents or an improvement in the liver histology of NASH patients [36].

\section{Angiotensin receptor blockers in cases of co-morbid hypertension}

Angiotensin II type I receptors are expressed on activated HSCs. Therefore, angiotensin receptor blocker (ARBs) may have antifibrotic effects in NASH. In an animal study, olmesartan improved fibrosis in NASH [37]. In a study of 54 NASH patients, telmisartan improved insulin resistance and NASH scores compared to valsartan [38]. A losartan study in NASH patients failed because of slow recruitment of patients due to the already-widespread use of ARB in NASH patients [39].

\section{New drugs under clinical trial}

OCA, selonsertib, CVC, and elafibranor are in phase 3 trials. Emricasan, aramchol, simtuzumab, NGM282, and BMS-986036 trials have been completed or are in phase 2 trials [40].

\section{Farnesoid X receptor agonist: obeticholic acid}

OCA is a first-in-class selective FXR agonist. Its mechanism of action scheme is shown in Fig. 6. In an animal model, OCA improved insulin sensitivity, glucose and lipid metabolism, and showed anti-inflammatory and anti-fibrotic effects in hepatic, renal, and intestinal tissues [41]. Two major phase 2 clinical trials were conducted to evaluate the safety and efficacy of OCA in biopsy-proven NASH patients $[41,42]$. In the FLINT phase $2 b$ trial ( $n=283), 45 \%$ of the patients in the 72 -week OCA group achieved the primary outcome (a decrease in NAFLD activity score by $\geq 2$ points without worsening of fibrosis) compared to $21 \%$ in the placebo group ( $p=0.0002)$. Fibrosis improvement was observed in $22 \%$ of the patients in the OCA group compared to $13 \%$ in the placebo group $(p=0.08)$. The main adverse events of OCA were increased low-density lipoprotein (LDL) cholesterol, decreased high-density lipoprotein cholesterol and pruritus (23\%) [42]. The CONTROL trial (NCT02633956) combining statins is ongoing. Two international phase 3 trials, RENERATE (NCT02548351) and REVERSE (NCT03439254) are now ongoing [41].

\section{ASK1 inhibitor: selonsertib}

ASK1 activation by mitochondrial oxidative stress, then JNK activation, is an important process in insulin resistance and inflammation [43]. The schematic mechanism of action is shown in Fig. 4. Selonsertib, an ASK1 inhibitor, significantly improved, not only metabolic parameters but also histologic parameters, such as hepatic steatosis, inflammation, and fibrosis [14]. In a 24 week clinical trial with or without simtuzumab, 43\% (13/30) of the patients in the $18 \mathrm{mg}$ selonsertib group showed reduced fibrosis ( $\geq$ one stage) compared with $30 \%(8 / 27)$ in the $6 \mathrm{mg}$ selonsertib group and $20 \%(2 / 10)$ in simtuzumab-alone group [14]. Two phase 3 clinical trials, STELLAR-3 (NCT03053050) and STELLAR-4 (NCT03053063), are now ongoing [40]. Common adverse effects are headache, nausea, fatigue, and upper abdominal pain [14].

\section{CCR2/CCR5 dual inhibitor: cenicriviroc}

The role of CCR2 and CCR5 for NASH progression are described in the introduction section. CVC is a potent inhibitor of CCR2/CCR5. In the phase 2b CENTAUR trial $(n=289)$, CVC did not meet the primary endpoint ( $\geq 2$-point NAS improvement or NASH resolution) but showed $\geq$ one stage fibrosis improvement after 1 year of treatment (20\% vs. $10 \%$ in the placebo group, $p=0.023)$. In contrast, CVC did not change body weight, aminotransferase levels, or insulin resistance. It was safe and tolerable, especially in terms of infection concerns [8]. The large phase 3 AURORA trial (NCT03028740) was initiated based on the efficacy and safety data of the CENTAUR trial $[7,40]$. About 2,000 patients were randomized 2:1 (CVC $150 \mathrm{mg}$ or placebo) to evaluate liver fibrosis improvement. They will undergo three consecutive liver biopsies (baseline, after 1 and 5 years). The trial will end in 2019. FDA approval might be determined by the outcome of the trial [40].

\section{PPAR $\alpha / \mathcal{S}$ agonist: elafibranor (GFT505)}

PPARs are composed of three isoforms: $\alpha, \beta / \delta$, and $\gamma$. PPARs are expressed in many tissues but differently distributed between the isoforms. For example, the $\alpha$ isoform is mainly in the liver and skeletal muscles, while the $\delta$ isoform is found in all tissues. PPARs participate in fatty acid oxidation and energy balance. Elafibranor (GFT505), a dual PPAR $\alpha / \delta$ agonist, improved plasma lipids and glucose homeostasis, insulin resistance, and reduced liver inflammatory markers [44]. The phase 2b GOLDEN-505 trial (NCT0164849) was conducted to evaluate the safety and efficacy of elafibranor. A total of $276 \mathrm{NASH}$ patients without cirrhosis were randomized to three groups (120 mg, $80 \mathrm{mg}$, and placebo). The primary endpoint (NASH reversal without progression of fibrosis at 52 weeks) was not met but post-hoc analysis based on a modified definition of response (disappearance of ballooning with the disappearance or mild persistence of lobular inflammation and no worsening of fibrosis), showed significant superiority in 
the 120 mg group. Liver enzymes, LDL-cholesterol, HbAlc, and inflammatory markers were significantly reduced in the $120 \mathrm{mg}$ elafibranor group. It was tolerated and safe, even though it caused a mild, reversible increase in serum creatinine [44]. The phase 3 RESOLVE-IT trial (NCT02704403) began in 2016 with the goal of recruiting 200 patients. Active recruitment will be completed in Dec 2021 [40].

\section{Pan-caspase inhibitor: emricasan}

Caspases are key enzymes in the apoptotic pathway. The detailed mechanism of action is shown in Fig. 2. Emricasan is a pancaspase inhibitor which blocks apoptosis. In phase 2 clinical trial (NCT02077374), 28-day emricasan therapy decreased ALT, cytokeratin 18 , and caspase $3 / 7$ significantly. It was safe and well-tolerated [45]. In the clinical study of 23 patients with compensated cirrhosis, 28-day emricasan treatment decreased portal pressure in a subgroup of patients with severe portal hypertension (hepatic venous pressure gradient $\geq 12 \mathrm{mmHg}$ ) [46].

\section{SCD1 modulator: aramchol}

Stearoyl-coenzyme A desaturase 1 (SCD1) is a key enzyme which converts saturated fatty acid (SFA) to monounsaturated fatty acid (MUFA). SCD1 expression results in MUFA formation. In contrast, its deficiency results in SFA accumulation. Overaccumulation of SFA may result in ER stress and apoptosis [2]. Aramchol, a conjugate of cholic acid and arachidonic acid, is an inhibitor of SCD1. In a phase 2 clinical trial $(n=58$, NCT01094158), $300 \mathrm{mg}$ aramchol treatment for 3 months decreased liver fat content and increased adiponectin levels significantly. Aramchol was safe and tolerable at a $300 \mathrm{mg}$ dose [47]. Further data are lacking.

\section{Monoclonal LOXL2 antibody: simtuzumab (GS-6624)}

The lysyl oxidase (LOX) family is composed of four isoforms, LOX and the LOXL1-4. Among them, LOXL2 is a key contributor to collagen crosslinking stabilization. Moreover, LOX2 promotes hepatic progenitor cells towards the cholangiocyte lineage, while suppressing their differentiation into hepatocytes (Fig. 5) [15]. Theoretically, blocking LOX2 activity attenuates collagen crosslinking and fibrosis, and promotes liver regeneration. Based on this background, a phase $2 \mathrm{~b}$ clinical trial of simtuzumab, an anti-LOXL2 monoclonal antibody, was conducted. Unfortunately, simtuzumab was ineffective in decreasing collagen content or the hepatic venous pressure gradient (NCT01672879) [16].

\section{Fibroblast growth factor 19 agonist; NGM282}

NGM282 is a variant of FGF-19, which reduces steatosis and lipotoxicity. In a phase 2 trial of 82 biopsy-proven NASH patients, $79 \%$ of the treatment group achieved the primary endpoint $(\geq 5 \%$ reduction in absolute liver fat content by magnetic resonance imaging-proton density fat fraction after 12 weeks of treatment) compared to $7 \%$ in the placebo group (NCT02443116). ALT level decreased and LDL cholesterol was increased in the treatment group [48].

\section{FGF-21 pegylated analogue; BMS-986036}

BMS-986036 (Pegbelfermin) is a pegylated analogue of FGF21. BMS-986036 decreased hepatic steatosis, NAFLD activity score (NAS) and fibrosis in a mouse NASH model, and improved insulin sensitivity, lipid profiles and fibrotic markers in obese diabetic patients. In a phase $2 \mathrm{a}$ trial of 75 obese biopsy-proven NASH patients, pegbelfermin achieved the primary endpoint and was tolerated during 16 weeks of treatment. The absolute hepatic fat fraction decreased $6.8 \%$ in the group who received a daily injection of $10 \mathrm{mg}$ pegbelfermin, $5.2 \%$ in the $20 \mathrm{mg}$ weekly injection group, and $1.3 \%$ in the placebo injection group ( $p=0.0004,0.008$, respectively). Pegbelfermin had beneficial effects on adiponectin levels, lipid profiles, aminotransferase levels, serum pro-C3, and liver stiffness [49].

\section{Conclusion}

The pathophysiology of NASH progression is complex. Therefore, one drug targeting a single pathway may not be effective. That is the reason why many drugs failed in clinical trials. Although several drugs succeeded in phase 2 trials and moved on to phase 3 , the efficacies were modest. Therefore, further research may be focused on combined therapy with two or more drugs covering different mechanisms of action.

\section{Conflicts of interest}

No potential conflicts of interest relevant to this article was reported.

\section{ORCID}

In Cheol Yoon, https://orcid.org/0000-0003-4150-4216

Jong Ryeol Eun, https:/ / orcid.org/0000-0002-8583-0737 


\section{References}

1. Oh H, Jun DW, Saeed WK, Nguyen MH. Non-alcoholic fatty liver diseases: update on the challenge of diagnosis and treatment. Clin Mol Hepatol 2016;22:327-35.

2. Alkhouri N, Dixon LJ, Feldstein AE. Lipotoxicity in nonalcoholic fatty liver disease: not all lipids are created equal. Expert Rev Gastroenterol Hepatol 2009;3:445-51.

3. Malhi H, Gores GJ. Cellular and molecular mechanisms of liver injury. Gastroenterology 2008;134:1641-54.

4. Yang L, Roh YS, Song J, Zhang B, Liu C, Loomba R, et al. Transforming growth factor beta signaling in hepatocytes participates in steatohepatitis through regulation of cell death and lipid metabolism in mice. Hepatology 2014;59:483-95.

5. Okazaki I. [Non-alcoholic steatohepatitis]. Cho YK, Eun JR, translators. Paju: Koonja Publishing Inc.; 2018.

6. Miura K, Yang L, van Rooijen N, Ohnishi H, Seki E. Hepatic recruitment of macrophages promotes nonalcoholic steatohepatitis through CCR2. Am J Physiol Gastrointest Liver Physiol 2012;302:G1310-21.

7. Tacke F. Cenicriviroc for the treatment of non-alcoholic steatohepatitis and liver fibrosis. Expert Opin Investig Drugs 2018;27:301-11.

8. Friedman S, Sanyal A, Goodman Z, Lefebvre E, Gottwald M, Fischer L, et al. Efficacy and safety study of cenicriviroc for the treatment of non-alcoholic steatohepatitis in adult subjects with liver fibrosis: CENTAUR Phase $2 \mathrm{~b}$ study design. Contemp Clin Trials 2016;47:356-65.

9. Paradis V, Perlemuter G, Bonvoust F, Dargere D, Parfait B, Vidaud $\mathrm{M}$, et al. High glucose and hyperinsulinemia stimulate connective tissue growth factor expression: a potential mechanism involved in progression to fibrosis in nonalcoholic steatohepatitis. Hepatology 2001;34:738-44.

10. Starley $\mathrm{BQ}_{2}$ Calcagno CJ, Harrison SA. Nonalcoholic fatty liver disease and hepatocellular carcinoma: a weighty connection. Hepatology 2010;51:1820-32.

11. Derosa G. Efficacy and tolerability of pioglitazone in patients with type 2 diabetes mellitus: comparison with other oral antihyperglycaemic agents. Drugs 2010;70:1945-61.

12. Sanyal AJ, Chalasani N, Kowdley KV, McCullough A, Diehl AM, Bass NM, et al. Pioglitazone, vitamin E, or placebo for nonalcoholic steatohepatitis. N Engl J Med 2010;362:1675-85.

13. Schuster S, Feldstein AE. NASH: novel therapeutic strategies targeting ASK1 in NASH. Nat Rev Gastroenterol Hepatol 2017;14:329-30.

14. Loomba R, Lawitz E, Mantry PS, Jayakumar S, Caldwell SH, Arnold H, et al. The ASK1 inhibitor selonsertib in patients with nonalcoholic steatohepatitis: a randomized, phase 2 trial. Hepatology 2018;67:549-59.

15. Ikenaga N, Peng ZW, Vaid KA, Liu SB, Yoshida S, Sverdlov DY, et al. Selective targeting of lysyl oxidase-like 2 (LOXL2) suppresses hepatic fibrosis progression and accelerates its reversal. Gut 2017;66:1697-708.

16. Harrison SA, Abdelmalek MF, Caldwell S, Shiffman ML, Diehl AM, Ghalib R, et al. Simtuzumab is ineffective for patients with bridging fibrosis or compensated cirrhosis caused by nonalcoholic steatohepatitis. Gastroenterology 2018;155:1140-53.

17. Keely SJ, Walters JR. The farnesoid X receptor: good for BAD. Cell Mol Gastroenterol Hepatol 2016;2:725-32.

18. Mudaliar S, Henry RR, Sanyal AJ, Morrow L, Marschall HU, Kipnes M, et al. Efficacy and safety of the farnesoid $\mathrm{X}$ receptor agonist obeticholic acid in patients with type 2 diabetes and nonalcoholic fatty liver disease. Gastroenterology 2013;145:574-82.

19. Grygiel-Górniak B. Peroxisome proliferator-activated receptors and their ligands: nutritional and clinical implications--a review. Nutr J 2014;13:17.

20. Miyahara T, Schrum L, Rippe R, Xiong S, Yee HF Jr, Motomura $\mathrm{K}$, et al. Peroxisome proliferator-activated receptors and hepatic stellate cell activation. J Biol Chem 2000;275:35715-22.

21. van Wagner LB, Rinella ME. The role of insulin-sensitizing agents in the treatment of nonalcoholic steatohepatitis. Therap Adv Gastroenterol 2011;4:249-63.

22. Bugianesi E, Gentilcore E, Manini R, Natale S, Vanni E, Villanova N, et al. A randomized controlled trial of metformin versus vitamin $\mathrm{E}$ or prescriptive diet in nonalcoholic fatty liver disease. Am J Gastroenterol 2005;100:1082-90.

23. Chalasani N, Younossi Z, Lavine JE, Diehl AM, Brunt EM, Cusi K, et al. The diagnosis and management of non-alcoholic fatty liver disease: practice guideline by the American Association for the Study of Liver Diseases, American College of Gastroenterology, and the American Gastroenterological Association. Am J Gastroenterol 2012;107:811-26.

24. Armstrong MJ, Gaunt P, Aithal GP, Barton D, Hull D, Parker $\mathrm{R}$, et al. Liraglutide safety and efficacy in patients with nonalcoholic steatohepatitis (LEAN): a multicentre, doubleblind, randomised, placebo-controlled phase 2 study. Lancet 2016;387:679-90.

25. Wadden TA, Hollander P, Klein S, Niswender K, Woo V, Hale PM, et al. Weight maintenance and additional weight loss with liraglutide after low-calorie-diet-induced weight loss: the SCALE Maintenance randomized study. Int J Obes (Lond) 2013;37:1443-51. 
26. Milder TY, Stocker SL, Abdel Shaheed C, McGrath-Cadell L, Samocha-Bonet D, Greenfield JR, et al. Combination therapy with an SGLT2 inhibitor as initial treatment for type 2 diabetes: a systematic review and meta-analysis. J Clin Med 2019;8:pii: E45.

27. Sumida Y, Yoneda M. Current and future pharmacological therapies for NAFLD/NASH.J Gastroenterol 2018;53:362-76.

28. Shibuya T, Fushimi N, Kawai M, Yoshida Y, Hachiya H, Ito S, et al. Luseogliflozin improves liver fat deposition compared to metformin in type 2 diabetes patients with non-alcoholic fatty liver disease: a prospective randomized controlled pilot study. Diabetes Obes Metab 2018;20:438-42.

29. Ito D, Shimizu S, Inoue K, Saito D, Yanagisawa M, Inukai K, et al. Comparison of ipragliflozin and pioglitazone effects on nonalcoholic fatty liver disease in patients with type 2 diabetes: a randomized, 24-week, open-label, active-controlled trial. Diabetes Care 2017;40:1364-72.

30. Tomita K, Teratani T, Suzuki T, Shimizu M, Sato H, Narimatsu $\mathrm{K}$, et al. Free cholesterol accumulation in hepatic stellate cells: mechanism of liver fibrosis aggravation in nonalcoholic steatohepatitis in mice. Hepatology 2014;59:154-69.

31. Muir K, Hazim A, He Y, Peyressatre M, Kim DY, Song X, et al. Proteomic and lipidomic signatures of lipid metabolism in NASH-associated hepatocellular carcinoma. Cancer Res 2013;73:4722-31.

32. Filozof C, Goldstein BJ, Williams RN, Sanyal A. Non-alcoholic steatohepatitis: limited available treatment options but promising drugs in development and recent progress towards a regulatory approval pathway. Drugs 2015;75:1373-92.

33. Sanyal AJ, Abdelmalek MF, Suzuki A, Cummings OW, Chojkier M; EPE-A Study Group. No significant effects of ethyl-eicosapentanoic acid on histologic features of nonalcoholic steatohepatitis in a phase 2 trial. Gastroenterology 2014;147:377-84.

34. Scorletti E, Bhatia L, McCormick KG, Clough GF, Nash K, Hodson L, et al. Effects of purified eicosapentaenoic and docosahexaenoic acids in nonalcoholic fatty liver disease: results from the Welcome* study. Hepatology 2014;60:1211-21.

35. Wang X, Ren Q, Wu T, Guo Y, Liang Y, Liu S. Ezetimibe prevents the development of non alcoholic fatty liver disease induced by high fat diet in C57BL/6J mice. Mol Med Rep 2014;10:2917-23.

36. Loomba R, Sirlin CB, Ang B, Bettencourt R, Jain R, Salotti J, et al. Ezetimibe for the treatment of nonalcoholic steatohepatitis: assessment by novel magnetic resonance imaging and magnetic resonance elastography in a randomized trial (MOZART trial). Hepatology 2015;61:1239-50.
37. Hirose A, Ono M, Saibara T, Nozaki Y, Masuda K, Yoshioka A, et al. Angiotensin II type 1 receptor blocker inhibits fibrosis in rat nonalcoholic steatohepatitis. Hepatology 2007;45:1375-81.

38. Georgescu EF, Ionescu R, Niculescu M, Mogoanta L, Vancica L. Angiotensin-receptor blockers as therapy for mild-to-moderate hypertension-associated non-alcoholic steatohepatitis. World J Gastroenterol 2009;15:942-54.

39. McPherson S, Wilkinson N, Tiniakos D, Wilkinson J, Burt AD, $\mathrm{McColl} \mathrm{E}$, et al. A randomised controlled trial of losartan as an anti-fibrotic agent in non-alcoholic steatohepatitis. PLoS One 2017;12:e0175717.

40. Connolly JJ, Ooka K, Lim JK. Future pharmacotherapy for non-alcoholic steatohepatitis (NASH): review of phase 2 and 3 trials. J Clin Transl Hepatol 2018;6:264-75.

41. Abenavoli L, Falalyeyeva T, Boccuto L, Tsyryuk O, Kobyliak N. Obeticholic acid: a new era in the treatment of nonalcoholic fatty liver disease. Pharmaceuticals (Basel) 2018;11:pii: E104.

42. Neuschwander-Tetri BA, Loomba R, Sanyal AJ, Lavine JE, Van Natta ML, Abdelmalek MF, et al. Farnesoid X nuclear receptor ligand obeticholic acid for non-cirrhotic, non-alcoholic steatohepatitis (FLINT): a multicentre, randomised, placebocontrolled trial. Lancet 2015;385:956-65.

43. Imoto K, Kukidome D, Nishikawa T, Matsuhisa T, Sonoda K, Fujisawa K, et al. Impact of mitochondrial reactive oxygen species and apoptosis signal-regulating kinase 1 on insulin signaling. Diabetes 2006;55:1197-204.

44. Ratziu V, Harrison SA, Francque S, Bedossa P, Lehert P, Serfaty $\mathrm{L}$, et al. Elafibranor, an agonist of the peroxisome proliferatoractivated receptor- $\alpha$ and $-\delta$, induces resolution of nonalcoholic steatohepatitis without fibrosis worsening. Gastroenterology 2016;150:1147-59.

45. Shiffman M, Freilich B, Vuppalanchi R, Watt K, Chan JL, Spada A, et al. Randomised clinical trial: emricasan versus placebo significantly decreases ALT and caspase 3/7 activation in subjects with non-alcoholic fatty liver disease. Aliment Pharmacol Ther 2019;49:64-73.

46. Garcia-Tsao G, Fuchs M, Shiffman M, Borg BB, Pyrsopoulos N, Shetty K, et al. Emricasan (IDN-6556) Lowers portal pressure in patients with compensated cirrhosis and severe portal hypertension. Hepatology 2019;69:717-28.

47. Safadi R, Konikoff FM, Mahamid M, Zelber-Sagi S, Halpern M, Gilat T, et al. The fatty acid-bile acid conjugate Aramchol reduces liver fat content in patients with nonalcoholic fatty liver disease. Clin Gastroenterol Hepatol 2014;12:2085-91.

48. Harrison SA, Rinella ME, Abdelmalek MF, Trotter JF, Paredes $\mathrm{AH}$, Arnold HL, et al. NGM282 for treatment of non-alcoholic steatohepatitis: a multicentre, randomised, double-blind, 
placebo-controlled, phase 2 trial. Lancet 2018;391:1174-85.

49. Sanyal A, Charles ED, Neuschwander-Tetri BA, Loomba R, Harrison SA, Abdelmalek MF, et al. Pegbelfermin (BMS986036), a PEGylated fibroblast growth factor 21 analogue, in patients with non-alcoholic steatohepatitis: a randomised, double-blind, placebo-controlled, phase $2 \mathrm{a}$ trial. Lancet 2019;392:2705-17. 